\title{
Einführung: Erzählen, eine Ressource zur imagefördernden (Selbst)Darstellung von Unternehmen
}

„Wenn wir nicht über uns reden, reden andere über uns. Und das meistens nicht so, wie es uns lieb wäre.“

(B. U. Biere 1994: 25)

Erzählen ist als ein „anthropologisches Grundbedürfnis des Menschen“ (Nünning/Nünning 2002: 9) in den verschiedensten Kulturen und Epochen allgegenwärtig. In der Wissenschaft ist es mit dem narrative turn (Lahn/Meister 2013; Iserhagen 1999) in den 1970/80er-Jahren zu einem interdisziplinären Forschungsfeld avanciert (The Narrativist Turn in the Human Sciences, Kreiswirth 1994). Im Zuge der Erkenntnis, dass dem Erzählen eine zentrale Rolle zukommt, der Welt und dem eigenen Dasein Sinn abzugewinnen, Ereignisse in zeitliche und kausale Zusammenhänge einzubinden und so Kohärenz zu erzeugen (Heinen 2007), ist das Interesse daran geradezu inflationär gestiegen. Infolge unterschiedlicher Forschungsperspektiven auf das Untersuchungsobjekt, Erzählen' rücken verschiedene Phänomene und jeweils spezifische Merkmale des Erzählens in den Vordergrund. Einige dieser Merkmale deuten grundsätzlich an, welche Potenziale Erzählungen auch für Unternehmen bergen. Der vorliegenden Arbeit liegt deshalb die Hypothese zugrunde, dass Unternehmen Erzählungen im Internet zum Zweck der eigenen Außendarstellung veröffentlichen. Bestimmte Merkmale des Erzählens und von Geschichten bieten Ansatzpunkte, um zu beschreiben, wie Unternehmen sie für eine positive (Selbst)Darstellung ((S)D) fruchtbar machen. Das betrifft beispielsweise die in Erzählungen mitgeteilte Erfahrungshaftigkeit (experienciality, Fludernik 1996), die vom subjektiven Erleben der Erzähler/innen geprägt ist. Zum Tragen kommt dieses Phänomen vor allem in Mitarbeitererzählungen, in denen Angestellte von ihrer Tätigkeit im Unternehmen erzählen, und dabei die 
spezielle (S)D des Unternehmens mit dem Ausdruck ihrer positiven Emotionen, ihres Erlebens und ihrer Erfahrung im Arbeitsalltag belegen. In der konversationsanalytischen Erzähltextanalyse steht mit Labov (1972) die tellability im Mittelpunkt, die sich insbesondere durch bewertende Bemerkungen von Erzähler/innen (Evaluationen), auszeichnet. Dabei tragen Bewertungen in weiten Teilen grundlegende Aspekte der (S)D und das eben auch im Fall von Erzählungen rund um Unternehmen. Schmid (2014: 3) wiederum sieht die Veränderung eines inneren oder äußeren Zustands (Zustandsveränderung) als grundlegendes Merkmal einer Erzählung. Das Merkmal wird für die (S)D von Unternehmen beispielsweise dann relevant, wenn die Unternehmenskommunikation eine Erzählung aus einer möglichst neutralen Perspektive verfasst. Die Verfasser/innen können dann zur (S)D auf Sachverhaltsbenennungen in Form der Verbesserung eines äußeren Zustandes (z. B. Öffentlichkeitsarbeit im Rahmen eines sozialen Projekts) referieren. So rücken sich Unternehmen selbst unaufdringlich in ein positives Licht, ohne sich des Eigenlobs bezichtigen lassen zu müssen (Biere 1991: 28). Diese narrativen Merkmale deuten bereits an, welche Möglichkeiten Erzählungen Unternehmen bieten, um sich selbst darstellen zu können. Unter (Selbst)Darstellungen verstehe ich in diesem Kontext Selbst- und Fremdbeschreibungen sowie Bewertungen und Handlungen, mit deren Hilfe auf eine gewünschte Außenwirkung Einfluss genommen werden soll. In Erzählungen von und über Unternehmen sind dementsprechend Aussagen darüber eingearbeitet, was ein Unternehmen leistet, wie es sich sieht oder gesehen werden möchte. (S)D basieren auf einer Darbietung, in der ausgewählte Aspekte einer Entität markiert und mit Bedeutung aufgeladen werden. Sie fungieren als Bewertungsvorschläge für die Öffentlichkeit und sollen komplexitätsreduzierend wirken (angelehnt an Szyszka 2010: 104). Hinter solchen (S)D steht ein Selbstbild/Selbstverständnis, das einer vorangenommenen, als sozialerwünscht erachteten Erwartungshaltung einer Zielgruppe entspricht und mithilfe von Erzählungen intersubjektiv vermittelt werden soll, um so ein positives Bild in der Öffentlichkeit zu stärken. Da diese Aufgabe nicht die Entität Unternehmen selbst erfüllen kann, sondern Vermittler/innen (z. B. Autorenkollektive, Mitarbeiter/innen u. a.) und Repräsentant/innen innerhalb und außerhalb des Unternehmens damit beauftragt, steht das Determinans des Determinativkompositums, (Selbst)Darstellung' in Klammern. Da das Unternehmen jedoch als ,juristische Person“ (Gohr 2002: 136) fungiert und letzten Endes die Verantwortung für sämtliche Handlungen seiner Mitglieder in ihrer Funktion trägt, referiere ich im Folgenden mitunter auch auf das Unternehmen in der semantischen Rolle des Agens.

Die Gründe, warum (S)D für Unternehmen relevant sind, sind vielfältig. Unternehmen können damit ökonomische Ziele verfolgen oder unternehmerischen 
Abgrenzungs- und gesellschaftlichen Anpassungsprozessen unterliegen. Heutzutage sind Unternehmen aufgrund internationaler Vernetzungen und der damit verbundenen Angebotsvielfalt einem immer stärkeren Wettbewerbsdruck ausgesetzt. Unter diesen Voraussetzungen wird es für sie zunehmend schwieriger, sich von anderen Unternehmen zu differenzieren, damit externe Bezugsgruppen ${ }^{1}$ sie wahrnehmen und gegenüber anderen Unternehmen präferieren. Mithilfe von Geschichten versuchen sie deswegen nicht nur sich von anderen Unternehmen abzugrenzen und ihre Bezugsgruppen für sich zu interessieren, sondern sie an sich zu binden (Beyer 2018; Schach 2016; Burel 2016: 26; Herbst 2015). Eine weitere Herausforderung besteht für Unternehmen darin, dass sich Konsument/innen über das Produkt hinaus dafür interessieren, wie und nach welchen Maßstäben Unternehmen produzieren und agieren (Ettl-Huber 2014, Bischl 2000: 46, 49). Das bringt letztere in die Situation, ihre Unternehmensphilosophie, -kultur und -ziele den Stakeholdern nachvollziehbar und transparent machen zu wollen. Damit verbunden ist das stetige Ringen um die eigene Glaubwürdigkeit und das Vertrauen der Bezugsgruppen, das gerade in Zeiten von Wirtschaftskrisen und Unternehmensskandalen an Bedeutung erlangt. Unter dieser Perspektive sieht Hillmann (2017) Erzählungen in Funktion, da ,[d]as Image und die Reputation eines Unternehmens [...] nicht nur auf der Qualität ihrer Produkte und Dienstleistungen, sondern auch auf den Geschichten, die über sie erzählt werden"2 (Hillmann 2017: 127) beruhe. Im Zuge dieser Anforderungen - sich von der Masse abzuheben, Bezugsgruppen zu werben und zu binden sowie unternehmensspezifische Konzepte zu kommunizieren - spielt das positive Vorstellungsbild von einem Unternehmen in der Öffentlichkeit (positives Image) eine grundlegende Rolle. Jedoch sind die Vorgänge rund um Unternehmen sowie Unternehmen selbst komplex. Für Außenstehende ist es nicht selbstverständlich, Zusammenhänge aus den meist nur bruchstückhaft einsehbaren Vorgängen abzuleiten. Geschweige denn, dass sie daraus unbedingt immer die gewünschten Schlüsse im Sinne der Unternehmensführung ziehen. Das einführende Zitat von Biere - „,[w]enn wir nicht

1 ,Als Bezugsgruppen oder Stakeholder bezeichnet man jene Akteure oder Gruppen im internen und externen Umfeld von Organisationen, die vom Handeln der Organisation beeinflusst werden oder die umgekehrt die Zielerreichung der Organisation beeinflussen können“ (Phillips/Freeman 2010, nach Zerfaß/Pleil 2015: 45).

${ }^{2}$ Als Hinweis zur begrifflichen Unterscheidung von Image und Reputation: „Image ist das spontane, intuitive Bild eines Unternehmens bei einzelnen Zielgruppen, während die Reputation eine meist langfristig orientierte, aggregierte Bewertung von Unternehmenseigenschaften und -handlungen in der Öffentlichkeit darstellt.“ (Mast 2015: 7, Hervorhebung, U.A.) Auf die Verwendung des Schlüsselbegriffs,Image“ gehe ich im folgenden Unterkapitel dezidierter ein. 
über uns reden, reden andere über uns. Und das meistens nicht so, wie es uns lieb wäre" (Biere 1994: 25) - kann ebenfalls auf das Erzählen angewandt werden. Die davon abgeleitete Devise lautet:

Geschichten über Unternehmen kursieren in der Öffentlichkeit, ob sie von den Unternehmen selbst angestoßen werden oder nicht. Solange Unternehmen von sich selbst erzählen lassen, können sie sich in den Geschichten so darstellen, wie sie in der Öffentlichkeit wahrgenommen werden wollen, denn auf Erzählungen anderer über sich haben Unternehmen - wenn überhaupt - nur sehr begrenzten Einfluss.

Erzählungen von und über Unternehmen können positive Vorstellungsbilder durch zwei Eigenschaften begünstigen: Zum einen können die Erzählungen so aufbereitet werden, dass sie die Komplexität der „Unternehmenswirklichkeit“ reduzieren, indem sie veranschaulichen, verständlich präsentieren und verifizierbar machen, wie abstrakt anmutende Konzepte innerhalb der Unternehmen umgesetzt und gelebt werden (z. B. Konzepte der Social Responsibility oder Diversity-Konzepte u. a.). Zum anderen stellen Unternehmen in den Erzählungen mithilfe ihrer positiven (S)D eine Bewertungsgrundlage für die Öffentlichkeit bereit. Das ist möglich, indem bestimmte Themen und Merkmale für die Geschichte ausgewählt, in den Erzählungen hervorgehoben und so mit Bedeutung aufgeladen werden.

Im Unternehmenskontext findet das Erzählen seit dem Einzug der um die 1999er-Jahre aus den USA eingeführten Storytelling-Methode großen Zuspruch. Die Idee des Storytelling hat ihren Ursprung in der Mitte der 90er-Jahre. Ursprünglich war die Methode als ein Instrument für das interne Unternehmensmanagement konzipiert (Thier 2006/2010). Heute ist der Begriff ,Storytelling' über die interne Unternehmenskommunikation hinaus auch in der externen Unternehmenskommunikation omnipräsent. Dabei ist Storytelling weder ein wissenschaftlicher noch ein „klar umrissene[r] und einheitlich definierte[r] Begriff" (Schach 2016: 11; Becker/Stude 2017: 1 f.). Jedoch ist zahlreichen Definitionen gemein, dass sie Erzählungen im Unternehmenskontext ein strategisches Kalkül zuschreiben: „Strategisch“ insofern, da diese Erzählungen auf bestimmte Wirkungsziele gerichtet sind. Auf diesem Weg soll eine kommunikative Einflussnahme auf einen anvisierten Adressatkreis ausgeübt werden (= instrumentelles Verständnis). Daher unterliegen sie übergeordneten Zielen der Unternehmenskommunikation (= Zweckgebundenheit), die beinhalten die Legitimation und/oder Reputation der Unternehmen zu erhöhen (vgl. Abschn. 7.1.1, 
Zerfaß/Pleil 2015; Röttger 2013). Entsprechend ist solch eine von der Unternehmenskommunikation ausgehende strategische Kommunikation abzugrenzen, von einer rein ,verständigungsorientierte[n] Kommunikation [...], deren Ziel die Herstellung eines rational motivierten Einverständnisses auf Baisis gemeinsamer Überzeugungen [ist]“ (Röttger et al. 2013: 11). Unter der strategischen Kommunikation von Unternehmen ist somit das Ankoppeln kommunikativer Aktivitäten an „die übergeordneten Ziele [...] des Unternehmens“ (Zerfaß/Pleil 2015: 45) zu verstehen. Vor diesem strategischen Hintergrund erzählen beispielsweise Unternehmensmitglieder Geschichten zur Vermittlung von Schlüsselinformationen und von Unternehmenszielen (Herbst 2011: 30; nach Schach 2016: 12). Darüber hinaus heißt es, dass Erzählungen Unternehmen die Option bieten, authentisch und emotional nach außen aufzutreten, um so von den Bezugsgruppen positiv erinnert zu werden (Hillmann 2017: 128). Ähnlich Vielversprechendes findet sich in der Praktikerliteratur. Neben dem Interessantheitskriterium und der damit einhergehenden Aufmerksamkeitsbindung sollen die Rezipient/innen in Erzählungen getroffene Aussagen besser nachvollziehen können ${ }^{3}$ und sie deswegen als glaubwürdiger erachten (Beyer 2018; Schach 2016, 2017; Rupp 2016; Mast 2015; Sammer 2015; Herbst 2015; Adamczyk 2015; Ettl-Huber 2014; Weinberg 2012; Denning 2011; Thier 2006/2010).

Die gerade vorgenommene Ausführung darüber, welche Stärken Erzählungen innerhalb der Unternehmenskommunikation zugeschrieben werden, zeigt, warum sich Unternehmen diese Stärken zu eigen machen wollen. Hierfür entwickeln von den Unternehmen beauftragte Agent/innen im Anwendungskontext narrative Praktiken. Der Begriff ,Praktiken“ wurde dabei aus zwei Gründen gewählt. Zum einen geht es darum, das vorgefundene Datenmaterial von dem inflationär verwendeten Terminus ,Storytelling ' abzugrenzen. Zum anderen zeigt das erhobene Korpus, dass das Abgrenzungsbedürfnis von Unternehmen gegenüber anderen Unternehmen häufig in einen kreativen und experimentellen Umgang mit dem Erzählen mündet, der im Anwendungskontext mal mehr, mal weniger oder gar nicht an narratologische Merkmale, Elemente und Muster von Erzählungen anknüpft, selbst wenn sie die Realisierung als ,Story“ oder ,Geschichte“ deklarieren. Unter der Bezeichnung ,narrative Praktiken' lassen sich so auch die weitaus häufiger vorgefundenen untypischen Realisierungen in die Untersuchung einbeziehen. Die Grundlage dafür ist die Spezifik von Praktiken, die

\footnotetext{
3 Teilweise kritisch zu den theoretisch hergeleiteten „Erfolgsversprechen“ positionieren sich Früh/Frey (2014) aufgrund empirisch erhobener Befunde.
} 
„durch den kontextsensitiven Einsatz von bestimmten sprachlich-kommunikativen Formen als Ressourcen zur Lösung grundlegender Aufgaben der Interaktionskonstitution und zur Herstellung bestimmter Handlungen [gekennzeichnet ist].“ (Schegloff 1997; Heritage 2010 nach Deppermann/Feilke/Linke 2016: 1)

Daraus folgt einerseits, dass Praktiken mit einem Kontext verbunden sind, der sie nicht nur rahmt, sondern auch hervorbringt. In dieser Arbeit sind das z. B. spezifisch pragmatische Aspekte wie institutionelle Vorgaben oder verfügbare Vermittler/innen, die die narrativen „Ergebnisse“ beeinflussen. Andererseits werden auf Basis der Optionen verschiedener Internet-Plattformen die möglichen Ressourcen ausgeschöpft, um die Aufgabe und Handlung der imagefördernden (S)D zu erfüllen. Daraus folgt, dass das aufgefundene Datenmaterial durch den Terminus ,narrative Praktiken' treffender erfasst wird, da so die kontexttypischen Realisierungen und nicht nur prototypische Erzählungen im narratologischen Sinne in die Analyse eingehen. Allerdings folgt daraus für die Untersuchung narrativer Praktiken eine holistische Perspektive auf die Daten. Im Hinblick auf den Kontextbezug bedeutet das die Reflexion einflussnehmender interdisziplinärer Erkenntnisse. In Bezug auf die linguistische Analyse folgt daraus, jene Gestaltungsmittel zu berücksichtigen, die für den Untersuchungsgegenstand konstitutiv sind (schriftliche und mündliche Erzähltexte, Bild, Bewegtbild, Ton, Musik u. a.). Unter diesen Voraussetzungen betrachte ich diese narrativen Praktiken als eine Ressource für Unternehmen, der Öffentlichkeit ein idealisiertes Selbstbild zu offerieren (Pätzmann 1993: 103, nach Vogel 2012: 103). Dabei zielen sie darauf, das Selbstbild möglichst schlüssig, wirklichkeitsnah und unterhaltsam zu gestalten.

Setzt man sich mit unternehmerischen $(S) D$ auseinander, kommt man nicht umhin, sich auch mit dem Thema Unternehmensidentität zu beschäftigen. Dabei sind zwei Begriffe als Hintergrundinformationen für diese Arbeit leitend: die Organisationsidentität und die Corporate Identity. Im Zuge der Erklärung, was sich dahinter verbirgt und inwiefern unternehmerische $(\mathrm{S}) \mathrm{D}$ damit in Beziehung stehen, wird ebenfalls der Begriff ,Image“ geschärft, damit sich die Fokussierung der vorliegenden Arbeit auf imagefördernde (S)D erschließt.

\subsection{Eine Perspektive auf die Identität von Unternehmen im Zusammenhang mit imagefördernden (Selbst)Darstellungen}

„Eine bewußte, gar strategische Selbstdarstellung bedarf psychologisch zwangsläufig eines Verständnisses des eigenen Selbst, es bedarf eines Selbst- oder 
Eigenbildes“ (Bungarten 1994: 8, Hervorhebung i. O.), das es im Rahmen der Identitätsarbeit herauszustellen gilt. Das Phänomen, dass Unternehmen über eine Identität verfügen, wird grundsätzlich nicht bestritten (Bischl 2000: 100). Sie ist sowohl für Außenstehende als auch für Unternehmensmitglieder wahrnehmbar. Allerdings besteht kein Konsens darüber, was genau darunter verstanden werden soll. Je nach Fachdisziplin und Fragestellung bestehen unzählige Ansätze. Daher greife ich zu den zwei ausgewählten Begriffen - Organisationsidentität und Corporate Identity - jeweils die Konzepte heraus, die ich als Hintergrundinformation zur Unternehmensidentität im Rahmen der Untersuchung als erhellend erachte ${ }^{4}$. Ich folge dabei einem betriebs- und wirtschaftswissenschaftlichen Ansatz, der die Unternehmensidentität als das Ineinandergreifen von Organisationsidentität und Corporate Identity sieht.

Grundsätzlich können die Identitäten von Individuen (Ich-Identität bzw. Individual-Identität), von Gruppen (Gruppen-Identität bzw. kollektive Identität) und von Organisationen (Organisations-Identität bzw. Institutions-Identität) unterschieden werden (Vogel 2012: 100). Hinsichtlich dieser theoretischen Unterscheidung darf jedoch nicht aus dem Blick geraten, dass die verschiedenen Identitätskonzepte miteinander in Beziehung stehen, - wie das später in der Erläuterung über die Corporate Identity ersichtlich werden wird. Doch bevor ein Konzept für identitätsbildende Prozesse im Spannungsfeld der verschiedenen Identitäten betrachtet werden kann, ist zu klären, wie sich das Phänomen ,Organisationsidentität' allgemein fassen und im Kontext der Arbeit mit narrativen (S)D verbinden lässt. Hierfür beziehe ich mich auf Eisenegger (2015). Er definiert die Identität von Organisationen unter Rückgriff auf den semiotischen Identitätsansatz von Christensen/Askegaard (2001). Die semiotisch fundierte Definition organisationaler Identität bezieht neben der Sprache noch weitere Zeichen ein, mittels derer Organisationen auf sich verweisen. Die Grundlage für das Modell ist der semiotische Prozess der Sinnbildung nach Preice. Die Unterscheidung zwischen dem Zeichen, einem Interpretanten und einem Objekt/Referent ist leitend für den Prozess der Sinnbildung - bezogen auf die Konstitution der organisationalen Identitätsbildung. Daraus ergibt sich folgende Adaption: Ein Zeichen steht für oder repräsentiert ein Objekt oder eine/n Referent/in. Das Objekt ist in dem Kontext die Organisation, während das Zeichen ,alle möglichen Formen organisationaler Repräsentationen sein können“ (Eisenegger 2015: 135). Im

\footnotetext{
${ }^{4}$ Eine Übersicht über den Diskurs sprengt den Rahmen der Arbeit und zielt am erzähltheoretischen Themenbereich vorbei. Ausführlichere Darstellungen bieten Eisenegger 2015, Rometsch 2008 oder Hatch/Schulz 2000.
} 
Prozess der Wahrnehmung erzeugen diese Zeichen bestimmte Vorstellungsbilder und Assoziationen (= Interpretant) im Zusammenhang mit der Organisation (= Objekt/Referent). (Christensen/Askegaard 2001: 305) Ausgehend von diesem Grundgedanken formuliert Eisenegger folgende Definition für die Identität von Organisationen:

„Die Organisationsidentität [...] ist die Summe aller organisationalen Signalisationen in Form von geplanten wie ungeplanten Selbstbeschreibungen einer Organisation. Diese Selbstbeschreibungen leiten die Wahrnehmung (= Image- und Reputationsbildung [...]) der Organisation als einer unterscheidbaren Größe mit charakteristischen Wesensmerkmalen sowohl organisationsintern als auch -extern an." (Eisenegger 2015: 436; Hervorhebung U.A.)

Die Organisationsidentität entsteht nach dieser Definition aus der Summe sämtlicher Zeichen, die das Objekt ,Organisation" vermittelt. Die Definition von Eisenegger (2015) über die organisationale Identität richtet sich vor allem an der Perspektive der Organisation auf die eigene Identität aus, da der Autor schreibt, dass sie durch ,die Summe aller organisationalen Signalisationen in Form von [...] Selbstbeschreibungen“ (ebd. 2015: 436) hervorgerufen wird. Zugleich wird darüber deutlich, dass narrative (S)D als Signalisationen einer Organisation einen Teilaspekt ihrer Gesamtidentität darstellen ${ }^{5}$. Dafür werden in Erzählungen spezifische Unternehmensmerkmale herausgestellt, die die Führungsebene als

„darstellenswert und mitteilenswert erachtet und von denen sie annimmt, daß sie den Erwartungen der Rezipienten an ein „gutes“, ,achtenswertes“ Unternehmen entsprechen“" (Bischl 2000: 108).

Somit handelt es sich bei den hier untersuchten Erzählungen um geplante (öffentlich zugängliche und inszenierte) (S)D. In den meisten Definitionen wird Identität als eine kommunikativ konstruierte Größe benannt. So auch bei Bungarten (1993: 8), der Unternehmen als soziokulturelle Gebilde sieht, deren Identität sich sprachlich-kommunikativ manifestiert und die durch Kommunikation vermittelt und erlebt werden kann. Dabei ist zu beachten, dass nicht allein die kommunizierten Inhalte, sondern auch kommunikative Handlungen der (S)D dazu dienen,

\footnotetext{
${ }^{5}$ In der Fachliteratur wird vorgeschlagen, geplante Selbstbeschreibungen einer Organisation unter dem Begriff,Organisationsprofil ‘ als eine Teilmenge der Organisationsidentität (Eisenegger 2015: 436) zu fassen. Darunter würden auch narrative Inszenierungen fallen. Da das für das Forschungsvorhaben der vorliegenden Arbeit jedoch nicht von Bedeutung ist, gehe ich darauf nicht weiter ein.
} 
eine Identität auszubilden (dazu später mehr in Abschn. 1.2). Da die Identitätsbildung u. a. auf kommunikativen Prozessen wie Selbstbeschreibungen beruht, zieht insbesondere die Praktikerliteratur häufig den Rückschluss, Unternehmen könnten den Identitätsbildungsprozess steuern. Diese Auffassung ist kritisch zu hinterfragen. So ist es nicht gewährleistet, dass die Inhalte einer geplanten, strategisch durchdachten Selbstaussage wie gewünscht rezipiert werden. Der semiotische Grundgedanke erlaubt es, weitere Zeichen und Zeichenkombinationen der organisationalen Identitätsstiftung über sprachliche Zeichen hinaus einzubeziehen (Einbezug von Fotos, Mimik, Gestik u. a.). Innerhalb dieser Arbeit gewinnt der Aspekt beispielsweise an Bedeutung, wenn sich Mitarbeiter/innen im Rahmen von audiovisuellen Erzählungen zu nahezu einheitlichen Figuren stilisieren (= figurierte Erzähler/innen). Sie stehen dadurch stellvertretend für ihr Unternehmen und werden aufgrund des durchdachten Zusammenspiels in Form der sprachlichen Performanz, ihres erzählten Handelns, ihrer Kleidung u. a. zu organisationalen Repräsentant/innen oder besser gesagt: zu ,Singalisationen" im Dienste der Unternehmensidentität. Auch in Bezug auf die Frage nach der Wirklichkeitsabbildung in Erzählungen von und über Unternehmen verdeutlicht die Definition, dass die Unternehmensrealität aufgrund der symbolischen Repräsentation anhand verschiedener Zeichen nicht der direkten Wahrnehmung zugänglich ist. Das ist ein bedeutender Grundgedanke, da mittels narrativer Praktiken genau das Gegenteil vermittelt werden soll: nämlich ein direkter Zugang zu der Unternehmenswirklichkeit durch eine/einen betroffene/n Erzähler/in.

Die Wahrnehmung einer Organisation mit den ihr eigenen Merkmalen bezeichnet Eisenegger (2015) in seiner Definition konform zum kommunikations- und wirtschaftswissenschaftlichen Diskurs als Image. Hierbei ist zu beachten, dass der Terminus ,Image“ in zahlreichen Disziplinen Anwendung findet. Nach Holly (1998) wird er vor allem in drei Varianten verwendet:

„1) als Bezeichnung für eine innere Vorstellung im Sinne einer erinnerten oder erdachten Wahrnehmung; 2) zur Bezeichnung der Gesamtheit von Vorstellungen, die ein einzelner oder eine Gruppe von sich bzw. einem anderen, einer Gruppe oder einer Sache hat; 3) als Bezeichnung für das kollektive Bild von einer Person, Gruppe oder Sache in der Öffentlichkeit" (Holly 1998: 224).

Das Image als das Fremdbild, das Personen von einem Unternehmen haben, fällt in die dritte Bedeutungsvariante, die zusammen mit der zweiten , in die Standardsprache eingegangen ist" (Holly 1998: 224). Die kommunikations- und wirtschaftswissenschaftliche Sichtweise ist nicht mit dem aus der Soziologie adapierten face-Begriff von Goffman (häufig übersetzt als Image) zu verwechseln, 
auf den sich Linguist/innen insbesondere zur Analyse von Gesprächen beziehen. Das face entspricht bei Goffman nicht einem kollektiven Bild von etwas, sondern einem Selbstbild, welches die alltägliche Interaktion mit koordiniert: „Der Terminus Image kann als der positive soziale Wert definiert werden, den man für sich durch die Verhaltensstrategie erwirbt, von der die anderen annehmen, man verfolge sie in einer bestimmten Interaktion. Image ist ein in Termini sozial anerkannter Eigenschaften umschriebenes Selbstbild“ (Goffman 1973: 10, nach Knape 2009: 35, Hervorhebung U.A.). In der Konversationsanalyse wurden die Prozeduren des Imageaufbaus, ausgehend von dem Goffmanschen face-Begriff, weiter ausdifferenziert. Schuster (2000) hält als gemeinsamen Nenner für die konversationsanalytische Forschung fest:

„Image ist ein kontextgebundenes Selbstkonstrukt, das innerhalb einer Kommunikationssituation interaktiv hergestellt wird und genau die Aspekte des Selbst fokussiert, die in dieser Situation relevant sind und aus der Sicht des Individuums relevant sein sollten." (Schuster 2000: 593)

Im Mittelpunkt steht bei dieser Sichtweise die Funktion des Image für die Interaktionssituation (z. B. Imageverletzungen in der Kommunikationsform Streit). In Bezug auf Texte existiert kein vergleichbares Gegenstïck zu der konversationsanalytischen Verwendung, da sie sich von Gesprächen in zwei grundlegenden Bereichen unterscheiden. Zum einen in Bezug auf das interaktive Merkmal, da die Gesprächssituation zeitlich zerdehnt ist. Hierzu zeigen die vorliegenden Analysen auch nur eine geringe Interaktivität zw. den Verfasser/innen der Erzählungen und den Rezipient/innen. Zum anderen dadurch, dass der Imageaufbau im Unternehmenskontext aus rezeptionsstrategischen Überlegungen resultiert. Schuster kommt daher zu dem Schluss:

„Im Bereich der Unternehmenskommunikation und ihrer vornehmlich betriebswirtschaftlich und/oder soziologischen Erforschung hat ein Imagekonzept Goffmanscher Prägung keinen Platz.“ (Schuster 2000: 596, siehe hierzu auch Lasch 2015: 304)

Medienvermittelte Images in sprachlicher und bildhafter Form (Vogel 2014: 191) erfasst die linguistische Imageanalyse (LIma). Sie nimmt Ansätze der kgonitiven Linguistik, der qualitativen, pragma-semotischen Hermeneutik auf und führt sie in der linguistischen Diskursanalyse fort (vgl. Vogel 2010, 2014). Mithilfe der LIma können öffentliche Images (vgl. Vogel 2010) aufgedeckt werden. Öffentliche Images entstehen durch rekurrente Sprachgebrauchsmuster in den Medien, durch welche individuelle Wissensrahmen medial koorientert werden. Dahinter steht ein konstruktivistischer Grundgedanke. Nach diesem bringen wir unsere 
Welt nicht nur sprachlich zum Ausdruck, sondern konstruieren sie auch sprachlich, indem wir darüber „mentale Modelle“ und Stereotype bilden (vgl. Vogel 2014). Das ,öffentliches Image“ trennt Vogel (2010: 7) explizit von ,konstruierten Wissensrahmen einzelner Textproduzenten (z. B. Journalisten, Medienredakteure usw.) und Textrezipienten“. Er fasst öffentliche Images als ,,potentielle Wissensangebote in Diskursen“ (ebd.), welche durch ,medien- und ausdruckseitig wie einzeltextübergreifende Aussagesysteme“ (ebd.) entstehen. Fassbar werden sie mittels medienvermittelter Stereotypen, die die Perspektive auf ein Referenzobjekt prägen. Sprachliche Ausdrücke spielen hierbei ein große Rolle, da sie Spuren „,kognitiver Textarbeit [sind], in der sich ,Verstehen“ von und ,Kommunizieren“ in Ausdruckskomplexen als aktiver Prozess der Sinn- und Weltherstellung zeigt“" (Vogel 2010: 4). Mit Vogles Worten bedeutet das vereinfacht gesagt:

„Je nachdem, welchen sprachlichen oder bildhaften Ausdruck wir verwenden, wird der bezeichnete Sachverhalt (z. B. Akteure, Orte, Sachverhalte usw.) in einer je besonderen Weise perspektiviert oder sprachlich zubereitet.“ (Vogel 2014: 102)

Da die untersuchten Erzählungen aufgrund ihrer Eingrenzung auf den Tehmenereich ,Erzählen“ kein massenmediales Bild abgeben, weil sie von einer überschaubaren Gruppe von Personen realisiert und rezipiert werden, ist dieser Imagebegriff in Teilen mit dem Untersuchungsgegenstand vereinbar. Gemeinsam mit dem hier zugrunde gelegten betriebs- und wirtschaftswissenschaftlichen Imagebegriff ist der konstruktivistische Grundgedanke. Ein grundlegender Unterschied besteht darin - auch wenn wir es mit medienvermittelten Stereotypen zu tun haben (Typen der (Selbst)Darstellung, Kap. 8) -, dass diskurslinguistisch erhobene Stereotypen und mentale Modelle weit über das von Unternehmen kommunizierte Image hinausgehen, da sie auf die Grundlage sozialen Handlens im Diskurs (Vogel 2010: 3) abzielen. Daher folge ich in dieser Arbeit dem kontextbezogenen, betriebs- und wirtschaftswissenschaftlichen Begriff des Image als Fremdbild: Das ,zumeist stark vereinfachte, typisierte und mit Erwartungen und Wertvorstellungen verbundene Vorstellungsbild über [...] ein Unternehmen“ (Mast 2015: 7), welches im Idealfall das Selbstbild einer Organisation reflektiert (Sandhu 2014: 1163) und so den funktionalen - also nicht den diskursiven Aspekt - fokussiert.

Diesem Ansatz folgend handelt es sich bei der Frage, was das Image eines Unternehmens ist, zuerst um eine Frage der Perspektive auf die Organisation: Wird also die Sicht seitens der öffentlichen Rezeption auf eine Organisation eingenommen, handelt es sich um das Image (Mast 2015: 7, Eisenegger 2015: 439; siehe auch Burel 2012: 7, 2015: 73; Vogel 2012: 103; Hatch/Schultz 2002: 991; 
Bungarten 1993: 118). Das so bezeichnete Fremdbild von Rezipient/innenen ,ist das Ergebnis eines Eindrucks und entspricht der Gesamtheit der Vorstellungen, die jemand über ein Unternehmen besitzt“ (Bischl 2000: 103). Damit geht einher, dass ein Image keine stabile Größe ist. Es kann als ein geschaffenes Bild in Frage gestellt werden und durchaus auch empirisch falsch sein, da es auf einem Eindruck basiert, der täuschen kann (vgl. Bischl 2000: 104). Eisenegger sieht das Image als ein beitragendes Element zur Organisationsidentität und umgekehrt. Er differenziert in seiner Definition über die Organisationsidentität nicht zwischen organisationsintern und -extern wahrgenommenen charakteristischen Identitätsmerkmalen. Dadurch ist die Identität auch über das Image zugänglich. Die Beziehung zwischen der Organisationsidentität und dem -image sieht er als wechselseitig an. Da Unternehmen ihr Image beobachten, dient es ihnen als ein Spiegel (Symbolischer Interaktionismus), der den Prozess der Identitätsbildung unterstützt (Eisenegger 2015: 438). Aus diesem Grunde sind Erzählungen von Unternehmen an bestimmten Themen, mit bestimmten Akteuren und innergeschichtlich auftretenden Figuren sowie an bestimmten (S)D ausgerichtet. Imagefördernde Selbstbeschreibungen richten sich unter dieser Prämisse auch an der antizipierten Wahrnehmung des unternehmensexternen Umfeldes aus. Was sich in imagefördernden Erzählungen letztlich widerspiegelt, ist somit nicht das eigentliche Fremdbild, sondern ein gewünschtes Image, das auf der Darstellung von Merkmalen eines kommunizierten Selbstbildes beruht. Die vermittelten Merkmale stehen somit in einer Wechselbeziehung mit dem Image und können sowohl organisationsintern wie auch -extern wahrgenommen werden. Dafür aufgewendete Signalisationen fußen hierbei eben auch auf Beschreibungen von Organisationsmitgliedern aus den betroffenen Organisationen. Alle diese Aspekte stellen - wie eben beschrieben - relevante Hintergrundinformationen für die zu beschreibenden narrativen (S)D von Unternehmen dar.

Der hier zugrunde gelegte Ansatz für die Identität von Unternehmen geht von der Annahme aus, dass sich die Unternehmensidentität im Zusammenwirken der Organisationsidentität und der Corporate Identity herausbildet. Dabei wird die Interaktion zwischen der Individual-Identität und der Kollektiv-Identität zum Dreh- und Angelpunkt der narrativen (S)D: Da Unternehmen heute mehr denn je ihre Mitarbeiter/innen erzählen ,lassen“, tragen diese erheblichen Anteil an dem Identitätsbildungsprozess von Unternehmen. Die vorausgegangene Abhandlung zur Organisationsidentität und dem Image habe ich vorrangig ausgeführt, um identitätskonstitutive Aspekte für narrative (S)D darzulegen. Bei der Ausführung über das Konzept der Corporate Identity geht es mir darum, die Bedeutung der Akteure rund um die Erzählungen für den identitätsbildenden Prozess zu verdeutlichen. 
Häufig wird der Begriff Unternehmensidentität synonym mit dem Begriff Corporate Identity verwendet (Burel 2012). Im Gegensatz dazu sieht eine synthetische Perspektive auf die Unternehmensidentität die Corporate Identity als einen mitbestimmenden, auf die Unternehmensidentität einflussnehmenden Bereich $a^{6}$. Diesem Ansatz zufolge bildet sich die Unternehmensidentität durch die wechselseitige Einflussnahme von organisationaler Identität und Corporate Identity aufeinander sowie durch das Spannungsverhältnis zwischen Individual-Identität und kollektiver Identität heraus. Diesem Konzept zufolge stellt die Organisationsidentität die Mitarbeiterperspektive auf die Unternehmensidentität dar, während die Corporate Identity die Sicht der Vorstandsebene beschreibt. Basierend auf Bungartens (1993: 116) Erkenntnis, dass der Begriff Corporate Identity mal als Methode, mal als Ergebnis eines Prozesses und mal als Theorie zu einem schillernden Begriff geworden ist, hält Bischl (2000) fest:

\begin{abstract}
„Als kleinster gemeinsamer Nenner der verschiedenen Definitionen und Auffassungen kann Corporate Identity hier als Bezeichnung für einen von Unternehmen geplanten Darstellungsprozeß von sich selbst bezeichnet werden, der zu weiten Teilen kommunikativ realisiert wird und auf das Ziel ausgerichtet ist, eine möglichst einheitliche und zugleich unverwechselbare Unternehmenspräsentation zu sein, um bei anderen eine positive Einstellung zum Unternehmen zu evozieren.“ (Bischl 2000: 106)
\end{abstract}

Deswegen ist die Corporate Identity meist strategisch ausgerichtet und damit einhergehend normativ geprägt (Bischl 2000: 105; Vogel 2012: 109 ff.). Wie die Organisationsidentität, die Corporate Identity, die Individual-Identität und die kollektive Identität mit der Unternehmensidentität zusammenspielen, veranschaulicht Vogel (2012) in dem folgenden Schaubild (Abb. 1.1).

Im Mittelpunkt der Abbildung steht die Unternehmensidentität. Sie entsteht im Zusammenspiel von zwei Prozessen, die einerseits von der Unternehmensführung (rechte Seite der Abb.) und andererseits von den Mitarbeiter/innen (linke Seite der Abb.) ausgehen. Die rechte Seite der Abbildung zeigt den Vorgang auf der Ebene der Unternehmensführung. Hierbei konstruieren Vertreter/innen der Unternehmensführung nach strategischen Erwägungen ein Konzept, in das sie auch zu Teilen die Sichtweise der Mitarbeiter/innen auf die Unternehmensidentität aufnehmen (= Identifikation) und wiederum andere Aspekte davon ausschließen (= Exklusion). Das Ergebnis dieses Prozesses ist die vorgegebene Corporate Identity seitens der Unternehmensführung. Ebenso verhält es sich auf der linken Seite der Abbildung, die den identitätsbildenden Prozess der Mitarbeiter/innen zeigt. Auch diese entwickeln eine bestimmte Perspektive auf die Unternehmensidentität

${ }^{6}$ Beispielsweise in Vogel 2012; Rometsch 2008; Hatch/Schultz 2000; Plätzmann 1993. 


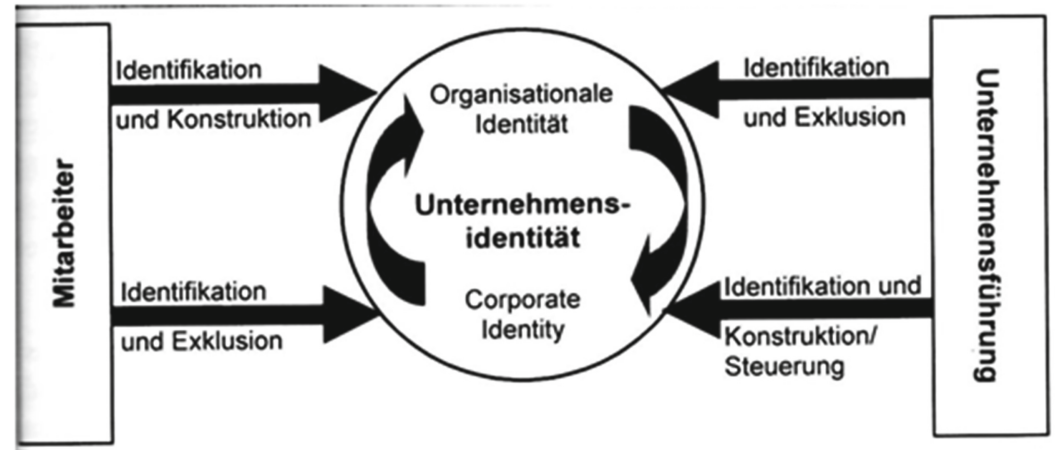

Abb. 1.1 Konstitution der Unternehmensidentität durch Mitarbeiter/innen und Unternehmensführung (Vogel 2012: 111)

(= Identifikation und Konstruktion). Dabei inkludieren und exkludieren sie ebenfalls Aspekte der von der Unternehmensführung vorgegebenen Corporate Identity. Daraus entwickelt sich wiederum die organisationale Identität ${ }^{7}$. Die Unternehmensidentität entsteht demnach aus der gegenseitigen Einflussnahme zwischen der organisationalen Identität und der Corporate Identity, deren Entstehungsprozesse ebenfalls durch die wechselseitige Perspektivenintegration miteinander verwoben sind. Das Modell zeigt, dass die Unternehmensidentität komplex ist. Sie geht über eine vorgegebene konstruierte Größe seitens der Vorstandsebene hinaus, indem sie auch die Mitarbeiter/innen in die Vorgänge einbezieht. Das ist u. a. eine Erklärung dafür, dass sich in dem erhobenen Korpus eine große Anzahl von Mitarbeitererzählungen befindet. Schließlich gestalten Mitarbeiter/innen nach diesem Modell die Unternehmensidentität mit und stehen als Repräsentant/innen für die Authentizität dieser Identität. In diesem Bereich kommt nun das Zusammenspiel von Individual-Identität und kollektiver Identität in den Blick. Da Unternehmen

\footnotetext{
${ }^{7}$ Eisenegger unterscheidet nicht zwischen der Sicht der Mitarbeiter/innen und der Führungsebene auf die Organisationsidentität. Er fasst darunter die „Innensicht“, die beide Seiten beinhaltet. Da sich die Organisationsidentität in diesem Modell dadurch etabliert, dass die Mitarbeiter/innen Aspekte der Führungsebene adaptieren, ist die Perspektive meines Erachtens mit der von Eisenegger kompatibel. Was dieses Modell im Gegensatz zu Eiseneggers Definition nicht zeigt, ist der semiotische Entstehungsprozess, der für diese Arbeit jedoch von Bedeutung ist. So dient jedes Modell für sich genommen dazu, aus seiner Perspektive jeweils grundlegende und konstitutive Merkmale der Unternehmensidentität aufzuzeigen, die für den Zusammenhang zwischen der (S)D von Unternehmen und der Unternehmensidentität relevant sind.
} 
eine kollektive Einheit darstellen, verfügen sie auch über eine kollektive Identität ${ }^{8}$. Diese kollektive Identität prägen die einzelnen Mitglieder einerseits mit ihren individuellen Merkmalen und andererseits, indem sie sich mit Merkmalen des Kollektivs identifizieren. Dadurch entsteht ein Spannungsfeld zwischen der Identität des Individuums und der Identität des Unternehmenskollektivs. Wenn nun Personen für das Unternehmen erzählen, müssen sie die Ambiguität zwischen ihrer Identität und der kollektiven Identität bewältigen. Das ist eine schwierige Aufgabe, da sie möglichst einheitlich adaptierte Merkmale der Corporate Identity kommunizieren sollen. Das sollen sie wiederum bewerkstelligen, ohne ihre Individual-Identität aufzugeben. Ansonsten stünde ihre Glaubwürdigkeit, aus ihrer persönlichen Wahrnehmung über das Unternehmen zu erzählen, infrage. Dieses Spannungsverhältnis ist für narrative Praktiken im Kontext von Unternehmen von großer Bedeutung.

(S)D von Unternehmen sind ein Teilaspekt einer gezielt nach außen getragenen Unternehmensidentität. Sie basieren auf Ergebnissen, die in einem komplexen Wechselspiel von Konstruktions- und Identifikationsprozessen der Mitarbeiter/innen und der Unternehmensführung entstehen. Dabei handelt es sich nicht um ein geschlossenes System, da Unternehmen ihre Außenwahrnehmung mittels eines erhobenen Fremdbildes ihrer Bezugsgruppen reflektieren und Schlüsse daraus ziehen, die sie wiederum in ihr Selbstbild integrieren. Das verdeutlicht, dass geplant und konstruiert imagefördernden (S)D im Unternehmenskontext Identitätsarbeit vorausgeht und - wie Eisenegger (2015) bereits betont -, die Beziehung zwischen der Unternehmensidentität und dem -image wechselseitig ist. Den Untersuchungsgegenstand bilden vor diesem Hintergrund Erzählungen, die ein positives Fremdbild in der Öffentlichkeit über das Unternehmen zu verstärken und zu festigen versuchen (= imagefördernd). Kommuniziert werden sollen auf diese Weise immaterielle Werte und keine Produkte. Das bedeutet jedoch nicht, dass in den Erzählungen keine Produkte der Unternehmen vorkommen können, schließlich sind sie ebenfalls Zeichen, die auf Unternehmen verweisen, und somit ein grundlegendes Identifikationsmerkmal - zumeist auch für erzählende Mitarbeiter/innen.

Imagefördernde (S)D in Erzählungen beruhen auf verschiedenen Zeichen, die mithilfe bestimmter narrativer Praktiken ansprechend und nachvollziehbar

\footnotetext{
${ }^{8}$ Von einer kollektiven Identität wird gesprochen, ,wenn diverse Individuen zusammenkommen und eine Gruppe (z. B. ein Unternehmen) bilden“ (Burel 2015: 61). Das Merkmal kollektiv kann auf Unternehmen angewandt werden, wenn Unternehmensmitglieder Selbstund Fremdzuschreibungen im Rahmen ihrer institutionellen Rolle treffen (ebd.).
} 
kommuniziert und medial vermittelt werden. Dabei spielt der Veröffentlichungsraum - das Internet - eine tragende Rolle, auf die ich im folgenden Unterkapitel eingehe.

\subsection{Das Internet: ein Kommunikationsraum für Erzählungen zur imagefördernden (Selbst)Darstellung von Unternehmen}

Die erhobenen narrativen Daten werden von Personen aus der Unternehmenskommunikation im Internet veröffentlicht. Durch das Internet können sich Computer nahezu weltweit miteinander vernetzen. ,[M]it der Entwicklung von HTTP (HyperText Transfer Protocols) für die Übertragung von Daten, des grafikfähigen HTML (Hypertext Markup Language), von Browsern wie Mosaic und des World Wide Web (WWW)“ (Marx/Weidacher 2014: 65) hat sich das Internet zu einem „Multimedium“ (Marx/Weidacher 2014: 72) entwickelt, das als eine „technische Plattform, [...] einen virtuellen Raum zur Kommunikation und zur Speicherung und Verbreitung von Informationen eröffnet“" (ebd. 2014: 82). Merkmale wie eine hohe Reichweite, geringe Kosten, ständige Verfügbarkeit, Schnelligkeit bei der Übertragung von Daten und die umfangreiche Erreichbarkeit von Zielgruppen begründen die große Attraktivität des Internets - auch oder gerade deswegen für Unternehmen. Nach der ARD/ZDF-Onlinestudie 2018 sind 90,3 Prozent der deutschsprachigen Bevölkerung ab 14 Jahren mit einer Nutzungszeit von 3:16 Stunden/Tag online (ARD/ZDF-Onlinestudie 2018). Unter den Nutzer/innen befinden sich verschiedene Zielgruppen, die Unternehmen mit einer geschickten Internetpräsenz online erreichen und auf sich aufmerksam machen können. Aus diesen Gründen ist das Internet heutzutage häufig der erste Kontakt zwischen Unternehmen und möglichen Interessenten (Nickl 2005: 121). Daraus resultiert, dass es ein wichtiger Faktor für die Öffentlichkeitsarbeit ist. So spricht beispielsweise Hillman (2017) davon, dass der Onlineauftritt auf der Unternehmenshomepage der Visitenkarte eines Unternehmens gleichkommt (Hillman 2017: 58).

Die rasante Entwicklung des Internets von einer Informationsquelle in Form von Websites, die nur von ihren Produzent/innen verändert werden konnten, hin zu einem Mitmach-Netzwerk - insbesondere im Zusammenhang mit dem Social 
Web - ist beachtlich ${ }^{9}$. Das Internet bietet hierfür einen virtuellen Kommunikationsraum, in dem sich Menschen auf den unterschiedlichen Plattformen über die Zeit und den Ort hinweg metaphorisch gesehen treffen, unterhalten und austauchen können. Damit einher gehen je nach Plattform spezifische Verwendungsregeln, die auf geteilten Normen, Erwartungen und Routinen über das angemessene Handeln auf der entsprechenden Plattform beruhen. Unabhängig vom Unternehmenskontext verweisen Untersuchungen darauf, dass die Kommunikation insbesondere im Social Web persönlicher, stärker informell sowie meinungsbetonter gestaltet ist (Mast 2016: 368; Schach 2016: 15; Tophinke 2009: 249). Auch Unternehmen sind sich bewusst darüber, „dass die Kommunikation im Netz ,anders“ ist" (Mast 2016: 375), als sie das aus ihrer Praxis mit den klassischen Medien (z. B. Zeitschriften, Fernsehen, Rundfunk u. a.) kennen. Trotz reichlich vorhandener Ratgeberliteratur, die Tipps und Tricks für die Kommunikation innerhalb der sozialen Medien bereithält, ist diese für Unternehmen eine Herausforderung, die sie nach Mast nur durch „Learning by doing“ (ebd. 2016: 376) bewerkstelligen können. Vor allem eine negative Reaktion auf veröffentlichte Inhalte und Aussagen in Form eines Shitstorms ist im Unternehmenskontext gefürchtet, da dieser zu einer eigenen Dynamik im Netz mit einem nennenswerten Imageschaden führen kann. Daher sind die untersuchten Unternehmen meines Erachtens im Internet - insbesondere im Social Web - auf ,leisen Sohlen“ unterwegs. Trotz der bestehenden „Fallstricke“ ist das Internet eine geeigneter Kommunikationsraum für Erzählungen, da Erzählen an sich in unserer Alltagspraxis ein persönlicher und informeller Vorgang ist, dem insbesondere die Umgebung im Social Web entspricht.

Hinzu kommt, dass das Internet als technische Basis die Grundlage für weitere Plattformen bildet, die ihrerseits den technischen Rahmen für die Anwendung verschiedener semiotischer Ressourcen vorgeben. Das bedeutet, dass es von der gegebenen Plattform abhängt, ob beispielsweise Fotos oder Filme darauf hochgeladen werden können oder wie der Text gestaltet werden kann u. a. Das führt dazu, dass die ,internetbasierten Kommunikationsumgebungen die Zunahme und Intensivierung von Multimodalität begünstigen“ (Stöckl 2016: 22; siehe auch

\footnotetext{
${ }^{9}$ In dem Prozess entwickelten sich die Akteure von einer passiv-konsumierenden Rolle hin zu einer aktiv-produzierenden Haltung (Runkehl 2014: 236). Dabei ist die Entwicklung nach Runkehl vor allem der Ausdruck des Willens und des Bedürfnisses der Teilnehmer/innen sich auszudrücken, mit anderen in Verbindung zu treten und Inhalte miteinander zu teilen (ebd. 2014: 236 f.). Lomborg (2014: 2) erweitert die kommunikativ aktiv-produzierende Haltung der Internetnutzer/innen dahingehend, dass diese auch in der Dimension der Software aktivproduzieren können.
} 
Marx/Weidacher 2014: 79; Androutsopoulos 2010: 433). So bietet das Internet Unternehmen vielfältige Möglichkeiten eine Geschichte zu präsentieren. Das kommt dem „Originalitätsdruck“, der auf der Unternehmenskommunikation vor allem bezüglich der sozialen Netzwerke lastet, entgegen, denn ,,[n]ichts langweilt Nutzer in den Social Media mehr, als dass Unternehmen bereits etablierte Ideen der Wettbewerber kopieren“ (Mast 2016: 376).

Nicht unerwähnt bleiben darf, dass die (S)D von Unternehmen im Internet nicht nur auf der Inhalts- und der Gestaltungsebene stattfindet, sondern auch auf der Handlungsebene. Also der Handlung, verschiedene Plattformen im Internet zu bespielen und zu pflegen. Darunter fallen zwei grundlegende Aspekte für imagefördernde (S)D: (a) Die Unternehmenskommunikation lässt sich auf das neue Kommunikationsangebot ein, sie „,geht sozusagen mit der Zeit“. (b) Je nach den technischen Möglichkeiten der bespielten Plattformen (z. B. Weblog, YouTube, Facebook u. a.) können Unternehmen eine Kommentarfunktion für ihre Bezugsgruppen freischalten oder sie in Aktionen dazu auffordern, selbst einen Erfahrungsbericht zu einem Thema (z. B. Recruiting-Veranstaltung, Gastbeiträge) zu verfassen. Damit nehmen sie das Prinzip auf, ihre Bezugsgruppen an der Kommunikation teilhaben zulassen (Beteiligungsprinzip). Insofern Unternehmen die Option realisieren, stellen sie sich im Lichte eines positiv bewerteten Kommunikationsverhaltens dar, ihre Bezugsgruppen nicht nur wahr, sondern auch ernst zu nehmen, um ihnen Wertschätzung und Bedeutung beizumessen.

\subsection{Erkenntnisinteresse und Aufbau der Arbeit}

Die Konzeption und Umsetzung der erhobenen Erzählungen beruht auf leitenden Erkenntnissen anderer Fachdisziplinen, deren Einbezug für die Analyse unumgänglich ist, um den Untersuchungsgegenstand adäquat zu fassen und $\mathrm{zu}$ beschreiben. Bei einer Analyse von unternehmerischen (S)D und ihrer narrativen Umsetzung gilt es nicht nur erzähltheoretische Aspekte, sondern ebenso Kontextfaktoren unter den sie prägenden betriebs-/wirtschaftswissenschaftlichen und sozialwissenschaftlichen Gesichtspunkten in der Untersuchung zu berücksichtigen. Ist es doch erst der spezifische Kontext, aus dem heraus sich dieses narrative Vermittlungsvorkommen entwickelt. Daher ist die Arbeit interdisziplinär ausgerichtet. Die konkreten narrativen und sprachlichen Realisierungen stehen dabei jedoch im Vordergrund und sind der Ausgangspunkt der explorativ qualitativ angelegten Untersuchungen, da (S)D nun mal in weiten Teilen mittels Kommunikation vermittelt werden. Insbesondere im Zusammenhang mit dem 
situativen Kontext und den erhobenen Grundtypen der (S)D stelle ich interdisziplinäre Bezüge her. Ursächlich dafür ist, dass ich es für sinnvoll erachte, die Analyseergebnisse mit konkreten einflussnehmenden Konzepten in den anwendungsbezogenen Gesamtzusammenhang zu bringen. Es ist nicht davon auszugehen, dass alle von Unternehmen beauftragte Personen solcher Erzählprojekte im Sinne von erzähltheoretischen Erwägungen agieren oder gar eine explizite erzähltheoretisch fundierte Ausbildung erfahren haben. Vor dem pragmatischen Hintergrund ist es naheliegend, dass die Ausführenden imagefördernde Aspekte in den Vordergrund stellen und die Erzählung eine „Verpackung“ darstellt, mit deren Hilfe die (S)D salonfähig werden sollen. Durch den Einbezug interdisziplinärer Erkenntnisse nehme ich die Frage nach dem ,Warum' in den Blick und trage der Entstehungssituation Rechnung. Erkenntnisleitend für die Analyse, vor der Haupthypothese, dass Erzählungen von Unternehmen im Internet funktional an ihrer (S)D ausgerichtet sind, sind zwei grundlegende Fragestellungen:

1) Welche Typen von (S)D setzen Unternehmen mithilfe von Erzählungen im Internet relevant?

2) Wie werden Erzählungen zur (S)D in dem situativen Kontext umgesetzt?

Das Ziel der Arbeit ist es - vor dem Hintergrund der pragmatisch ausgerichteten Forschungsfragen - theoretische und formale Aspekte von Erzählungen mit funktionalen Aspekten unternehmensbezogener Erzählungen zu verbinden. Der Weg führt dabei von der Erhebung spezifisch narrativer Realisierungen im Unternehmenskontext zu Aussagen über angewandte narrative Praktiken in ihrer Funktion, letztere ermöglicht auf die Bedeutung von Erzählungen für Unternehmen zur imagefördernden (S)D rückzuschließen. Damit hat die Arbeit einen unmittelbaren Anwendungsbezug. Für die Erzählforschung liegt das Interesse an der Analyse einerseits darin, dass es sich um Gebrauchstexte (Rolf [1993] 2015) in einem institutionellen Anwendungsbereich handelt, die aufgrund der Anforderungen eigene Charakteristika erwarten lassen. Andererseits liegt das Forschungsinteresse darin, spezifische Merkmale von Erzählungen vor den Möglichkeiten der digitalen Technologie im Internet zu reflektieren: Das Internet gibt bestimmte Randbedingungen vor, die sowohl Auswirkungen auf den Inhalt als auch auf die Produktionsstrategien haben (Nickl 2005: 129). In diesem Rahmen entwickeln sich narrative Umsetzungen, die zwischen bekannten Gattungen (z. B. Reportage) und experimentellen Versuchen (z. B. diskontinuierliche Erzählungen) oszillieren. Diese teils neuartigen und sehr heterog auftretenden Vermittlungsvorkommen gilt es hinsichtlich bestimmter Gemeinsamkeiten zu bündeln und zu beschreiben. Vor diesem Hintergrund stehen spezifische Unterfragen im Mittelpunkt des Interesses: 
- Inwiefern befördern Erzählungen imagefördernde (S)D?

- Welche Elemente sind für diese pragmatisch ausgerichteten Erzählungen konstitutiv?

- Werden zugunsten einer imagefördernden (S)D bestimmte narrative Elemente an den Bedarf adaptiert?

- Welche Merkmale sind für die Erzähler/innen und weitere involvierte Akteure in dem Kontext bezeichnend?

- Wie werden verschiedene semiotische Ressourcen für Erzählungen zur imagefördernden (S)D genutzt?

Ausgehend von der einleitend beschriebenen Situation erachte ich drei Einflussfaktoren auf diese Erzählungen von Unternehmen als bedeutend. Diesen Faktoren liegen drei Subhypothesen hinsichtlich der narrativen Gestaltung zugrunde, die in der vorliegenden Arbeit Berücksichtigung finden:

1. Der pragmatische Hintergrund der (S)D beeinflusst die Inhalte der Erzähltexte hinsichtlich der erzählten Ereignisse wie auch der Gewichtung spezifischer narrativer Elemente/Merkmale.

2. Die narrative (S)D von Unternehmen übernehmen Mittler/innen, die entweder Teil des Unternehmens oder von demselben beauftragt sind. Das beeinflusst die narrative Realisierung, die Auswahl sowie die Rollen möglicher textextern und textintern handelnder Akteure.

3. Der Realisierungskontext im Internet eröffnet verschiedene Optionen, die zur imagefördernden (S)D von Unternehmen genutzt werden: a) multimodale Ressourcen und b) unterschiedliche Kommunikationsräume.

Im Kontext von Unternehmen entstehen auf deren Internetplattformen kreativ narrative Gebilde, die aus narrativen Praktiken zur imagefördernden (S)D resultieren. Dabei bilden sich spezifische textexterne und -interne Akteure heraus, die in einem Spannungsfeld von Wirklichkeit und erzählerisch optimierter Wirklichkeit operieren müssen. Sie stehen vor der Herausforderung, so zu erzählen, dass sie sowohl normative Vorgaben (z. B. im Sinne der Corporate Identity) als auch persönliche Aspekte oder, anders gesagt, den Ist- mit dem Sollzustand in „Einklang“ bringen. Die Arbeit beinhaltet keine Rezeptionsstudie, sondern entspricht dem, was in der Medienwissenschaft eine Produktanalyse genannt und in der qualitativen Forschung als qualitative Inhaltsanalyse bezeichnet wird (Holly 2010: 362). In dem beschriebenen Rahmen bietet die Arbeit einen unmittelbaren Anwendungsbezug zu einem Trendthema, aus dem spezifische Erzählungen hervorgehen, deren Betrachtung sich in ihrem vielfältigen 
und vermehrten Vorkommen rechtfertigt. Die untersuchten (S)D resultieren vor den gegebenen theoretischen Erwägungen nicht aus der Produktwerbung, sondern beziehen sich auf die Kommunikation einer Unternehmensidentität, die das Unternehmen hinsichtlich seiner ethischen und moralischen Merkmale kennzeichnet (Unternehmensphilosophie, -werte, -kultur). Die Produktwerbung ist meines Erachtens ein eigener Untersuchungsbereich, den ich in diese Arbeit nur zur Spezifizierung des Untersuchungsgegenstandes einbeziehe.

Die Arbeit ist in zehn Kapitel gegliedert. Kapitel zwei hat zum Zweck, die materiale Untersuchungsgrundlage vorzustellen. Das Korpus wird anhand des Vorgehens zur Datenerhebung, der daraus resultierenden Materialsammlung und Archivierung sowie einem Überblick über die Häufigkeitsverteilung der Daten vorgestellt. Das Kapitel spiegelt dabei die heterogene Datenlage wider. Die Kapitel drei bis einschließlich fünf umfassen theoretische Hintergründe, auf deren Basis die Daten beleuchtet und eingeordnet werden.

In Kapitel drei beginne ich damit, den Untersuchungsgegenstand aus der linguistischen Perspektive zu spezifizieren. Das beinhaltet, dass ich das Untersuchungsobjekt textlinguistisch verorte (Abschn. 3.1). Im Weiteren ordne ich die Daten als eine Erscheinungsform der Textsortenklassifizierung ,Repräsentationstexte " (Gohr 2002) ein, da sie alle dem übergeordneten Prinzip folgen, ,im Dienst des Unternehmens zu stehen und dieses optimal zu repräsentieren" (Gohr 2002: 304 f.). Burel (2015: 150) sieht darüber hinaus die Bezeichnung im Zusammenhang mit der Unternehmensidentität als zielführend an, da Repräsentation auch die Vermittlung von „Wirklichkeit“ durch Zeichen beinhaltet (Abschn. 3.2). Die abschließende Spezifizierung behandelt den pragmatischen Aspekt in Form von Berühr- und Unterscheidungspunkten zur klassischen Werbung, ebenfalls aus der textlinguistischen Perspektive. Eine Form der „Eigenwerbung“ ist nicht zu leugnen, obwohl eine eindeutige Nähe zur Werbung für Erzählungen in dieser Funktion kontraproduktiv ist (Abschn. 3.3).

Im darauf folgenden Kapitel vier reflektiere ich Entwicklungen der linguistischen Erzählforschung. Dabei fokussiere ich mich auf jene Erkenntnisse, die in einem engen Zusammenhang mit dem Untersuchungsgegenstand stehen und die im Rahmen der folgenden Untersuchungen fruchtbar gemacht werden.

Das fünfte Kapitel leite ich ein, indem ich zentrale Begriffe, die im Diskurs rund um das Erzählen von Bedeutung sind, aufgreife. Damit ist die Frage eng verbunden, was eigentlich narrativ ist (Abschn. 5.1). Die Bearbeitung der Frage ist die Grundlage der folgenden Unterkapitel, in denen ich die Merkmale der erhobenen Erzählungen vor dem erzähltheoretischen Hintergrund darlege, der dadurch sozusagen in situ, auf die Arbeit zugeschnitten ist. Daraus resultiert ein Verortungsschema, das dazu dient, die voneinander unterschiedlichen Erzählungen 
mit ihrer narratologischen Spezifik zu erfassen. Ausgehend von der Grundformel von Martinez „Erzählen ist Geschehensdarstellung + x“ (ebd. 2017: 3) formuliere ich inhaltliche (Abschn. 5.2) und formale (Abschn. 5.3) Merkmale, die für die erhobenen Daten bezeichnend sind. Dabei beziehe ich mich sowohl auf die linguistische wie auch auf die literaturwissenschaftliche Forschung. Das ist in der neueren linguistischen Erzählforschung nicht mehr ungewöhnlich. So berufen sich beispielsweise in der Forschung zum Erzählen via Twitter einige Linguist/innen (z. B. Puschmann/Heyd 2012; Rinke 2012; Page 2015) auf erzähltheoretische Konzepte literaturwissenschaftlicher Provenienz. Das Kapitel schließt mit einem aus den vorausgegangenen Ausführungen entstandenen Schema, in dem sich diese speziellen Erzählungen zur imagefördernden (S)D narratologische verorten lassen (Abschn. 5.4).

In Kapitel sechs gehe ich darauf ein, welche methodischen Grundüberlegungen der qualitativen Forschung ich für die Analyse adaptiert habe und welche linguistischen Methoden ich einbeziehe. Dabei erläutere ich den theoretischen Rahmen der Methoden und ihre Eignung für das Forschungsinteresse. An das Kapitel schließen die so erzielten empirischen Analyseergebnisse an.

Kapitel sieben beschreibt den situativen Kontext der erhobenen Daten. Das Kapitel bezieht sich auf spezifische Einflussfaktoren, denen die konkrete Umsetzung der Erzählungen unterliegt. Da es sich um eine strategische, zielgerichtete Kommunikationsmaßnahme handelt, müssen sowohl bestimmende Einflussfaktoren von außen auf die narrativen Praktiken als auch Einflussfaktoren, die aufgrund der pragmatischen Ausrichtung mit den Inhalten der Erzählungen verbunden sind, angesprochen werden. Der situative Kontext teilt sich somit in diese zwei Bereiche auf: a) „text"-externe Produktionsbedingungen und b) „text"-interne Produktionsbedingungen. Das Ziel ist es, übergeordnete Rahmenbedingungen und Zusammenhänge $\mathrm{zu}$ erfassen und $\mathrm{zu}$ beschreiben, die die Erzählungen zur imagefördernden (S)D im Internet beeinflussen. „Text“-externe Produktionsbedingungen, die auf die Erzählungen wirken, entstehen aufgrund der Voraussetzungen, unter denen sie verfasst und veröffentlicht werden. Das betrifft die Verantwortlichen der Veröffentlichungen, die als Mitglieder der externen Unternehmenskommunikation für den speziellen Aufgabenbereich ,Öffentlichkeitsarbeit' (Public Relations) zuständig sind. Des Weiteren nehme ich in diesem Kapitel die Produzent/innen anhand des Konzepts der multiplen Autorenschaft in den Blick und wende mich danach dem Rezipientenkreis zu, der insbesondere durch das Modell der Mehrfachadressierung auf die Erzähltextkonzeption wirkt (Abschn. 7.1). Neben diesen „,text“-externen Produktionsbedingungen müssen die Verfasser/innen auch die pragmatische Ausrichtung auf imagefördernde 
(S)D berücksichtigen. Diesen Bereich nenne ich „text“-interne Produktionsbedingungen. Sie spiegeln sich in der Auswahl der Themenfelder und in der Auswahl des innergeschichtlich auftretenden Personals. Nicht jedes Thema ist automatisch für eine imagefördernde (S)D gewinnbringend. Dementsprechend kristallisieren sich in dem Datenmaterial bestimme Themenfelder mit einem dazugehörigen „Akteurskreis“ heraus, dem bestimmte Rollen und Funktionen in den Erzählungen zugeschrieben werden, die auf die imagefördernden (S)D zurückgehen (Abschn. 7.2).

In Kapitel acht behandle ich die Stilisierung von vier aus den Daten hergeleiteten narrativen Grundtypen der (S)D. Diese Grundtypen konstituieren sich mithilfe von Untergruppen, die ich als Facetten des Grundtyps erfasse. So können sich Unternehmen nach dem selben Grundtyp darstellen, indem sie unterschiedliche Facetten bedienen. Die Grundlage sind selbstdarstellende Äußerungseinheiten, die bestimmte Akteure auf Basis von bestimmten Sprechhandlungen treffen. Um nachvollziehbar $\mathrm{zu}$ machen, was Unternehmen dazu veranlasst, eine bestimmte $(S) D$ zu vollziehen, lade ich die einzelnen Grundtypen mit fachexternen Hintergrundinformationen auf. Im Anschluss daran folgen charakteristische Realisierungsmerkmale des jeweiligen Typen. Inwiefern der Kommunikationsmodus ,Erzählen' für diese Typenstilisierung fruchtbar gemacht wird, verdeutlicht die jeweils dafür herausgearbeitete narrative Rhetorik, deren Sinn und Zweck es ist, einen Grundtyp möglichst eingängig zu machen.

Kapitel neun beinhaltet ausgewählte narrative Vermittlungsvorkommen der erhobenen Erzählungen zur (S)D. Daher wird in diesem Kapitel nur situativ auf spezifische Aspekte zur imagefördernden (S)D hingewiesen. Dem Kapitel liegt die Frage zugrunde, welche spezifischen Merkmale solche Erzählungen im Ganzen kennzeichnen. Das ist von Interesse, da die erhobenen Daten sich aufgrund der unterschiedlichen Internetplattformen, auf denen sie stehen, verschieden voneinander präsentieren. Aufgrund des Untersuchungsinteresses bündel ich das äußerst heterogene Vermittlungsvorkommen anhand gemeinsamer Merkmale. Das betrifft vorrangig Beispiele zu Twitter (Abschn. 9.4) und Facebook (Abschn. 9.3). Der Umgang mit dem Erzählen auf diesen Plattformen ist nach wie vor noch experimentell. Daher sind diese Kapitel umfassender als die Kapitel über Blogerzählungen (Abschn. 9.2) und Gründungsgeschichten (Abschn. 9.1). Diese beiden Bereiche sind in den vergangenen fünf Jahren stärker in den Fokus der Forschung gerückt. Aus diesem Grund spiegle ich die Analyseergebnisse dieser beiden Kapitel anhand bestehender Forschungsergebnisse und fokussiere jeweils spezifische Merkmale, die in dem Korpus hervorstechen. Im Mittepunkt stehen vorrangig Merkmale der narrativen Struktur, der sprachlichen und multimodalen Realisierung sowie Maßnahmen der Erzähler/innen ihre Beteiligung 
am Geschehen (Involviertheit) zu verdeutlichen. Letzteres ist für die Glaubwürdigkeit der (S)D und Aufmerksamkeitsweckung der Rezipient/innen grundlegend, daher werden verschiedene Strategien aufgewendet, um den Eindruck eines hohen Maßes an Involviertheit der Erzähler/innen zu wecken. Veranschaulicht wird das narrative Vermittlungsvorkommen anhand der Internetplattformen, auf denen es überwiegend aufgefunden wurde.

In Kapitel zehn fasse ich abschließend, die zentralen Aspekte der Arbeit und der Analysen zusammen.

Abschließend folgen nun noch Hinweise zu formalen Umsetzungen: Die typografischen Zeichen im Schrifttext wende ich in den folgenden, tabellarisch aufgeführten Funktionen an (Tab. 1.1):

Tab. 1.1 Anwendung typografischer Zeichen

\begin{tabular}{l|l}
\hline Typografisches Zeichen & Verwendung \\
\hline Kursiv & $\begin{array}{l}\text { Fachausdrücke / Kapitelverweise / Aufsatz- und } \\
\text { Buchtitel }\end{array}$ \\
\hline Unterstreichungen & $\begin{array}{l}\text { Hervorhebung in: } \\
- \text { ausformulierten Aufzählungen } \\
- \text { Zitaten (sowohl Erzähl- wie auch Fachtextzitate) }\end{array}$ \\
\hline „doppelte Anführungszeichen“ & Zitate / sinnbildliche Ausdrücke \\
\hline ,einfache Anführungszeichen“ & Begriffe oder Phrasen auf die ich referiere \\
\hline kursiver Fettdruck & $\begin{array}{l}\text { Objektsprache: (Erzähl-)Zitate aus dem } \\
\text { Datenmaterial }\end{array}$ \\
\hline
\end{tabular}

Screenshots von Portraits von den Unternehmenswebsites sind aufgrund der Persönlichkeitsrechte nachgezeichnet worden. Gesichter in Filmtranskripten, deren Mimik nicht Bestandteil der Analyse ist, habe ich zum Zweck der Anonymisierung verpixelt. Den Text von Tweets oder Facebookposts habe ich ebenso zu Gunsten der Anonymisierung größtenteils abgeschrieben und für gewöhnlich ausschließlich beigefügte Fotografien als Screenshot übernommen.

Internetquellen belege ich im Fließtext anhand von Kurzformen, um den Lesefluss nicht zu beeinträchtigen. Die entsprechenden URL für wissenschaftliche Dokumente und zitierte Statistiken führe ich im Literaturverzeichnis auf. Beispiele aus den Datenkorpora zitiere ich mithilfe von Kurzzitaten. Die Internetquelle kann aufgrund einer eigenen Erzähl-ID im Anhang unter „Quellverzeichnis der Unternehmenszitat nach Korpusquelle: Erzähl-ID“ nachvollzogen werden. 
Der gesamte Zitatausweis für die zitierten Unternehmensdaten besteht aus zwei Teilen. Der erste Teil (= Zitationsverweis) verweist auf das Unternehmen, da ich die Daten (mit wenigen Ausnahmen) sowohl im Text als auch im Bild anonymisiert habe. Danach folgt die Internetplattform auf der die Erzählung erhoben wurde. Ist ein Veröffentlichungszeitpunkt benannt, bildet er das letzte Element des ersten Zitationsverweises. Andernfalls indiziert die Abkürzung ,o. J.' (ohne Jahres- / bzw. Datumsangabe), dass kein Veröffentlichungsdatum vermerkt wurde (bspw. häufig im Fall von Unternehmensgeschichten). In diesem Fall vermerke ich in dem angehängten „Quellverzeichnis der Unternehmenszitate“ das Datum des letzten Zugriffs. Entsprechend sieht der erste Teil des Zitationsverweises wie folgt aus:

\section{Erster Teil: Zitationsverweis}

[Unternehmen]_[Internetplattform]_[Veröffentlichungsdatum / o. J.]

Bsp. $\rightarrow$ E.ON, Facebook, 2016-02-23.

Der zweite Teil des Zitationsverweises beinhaltet, unter welcher Kennung die URL in dem „Quellverzeichnis der Unternehmenszitate“ zu finden ist. Die Kurzform für den Ausweis der Korpusdaten im Fließtext ist folgendermaßen aufgebaut:

\section{Zweiter Teil: Korpusquelle}

[Kürzel- oder Unternehmensname]_[laufende Kennungszahl im Korpus]_[Internetplattform]

$$
\text { Bsp. } \rightarrow \text { Korpusquelle: EDEKA_263_YT }
$$

Ein Beispiel für einen gesamten Kurzverweise wäre nach dem beschrieben Muster:

(Daimler, Facebook, 2016-08-06 / o. J. Korpusquelle: DAIMLER_121_FB)

Textuelle Screenshots, die also ausschließlich sprachlich realisiert sind, habe ich in den meisten Fällen abgeschrieben. Wenn ich dennoch ein Bildschirmfoto (z. B. wegen typografischer Merkmale), das allein sprachlich ausgeführt ist, eingefügt habe, fällt das nach Marx/Weidacher (2014: 21) nicht unter eine Abbildung und ist daher auch nicht als Abbildung beschriftet, sondern als Beispiel mit dem oben beschriebenen Zitationsverweis ausgezeichnet. 
Open Access Dieses Kapitel wird unter der Creative Commons Namensnennung 4.0 International Lizenz (http://creativecommons.org/licenses/by/4.0/deed.de) veröffentlicht, welche die Nutzung, Vervielfältigung, Bearbeitung, Verbreitung und Wiedergabe in jeglichem Medium und Format erlaubt, sofern Sie den/die ursprünglichen Autor(en) und die Quelle ordnungsgemäß nennen, einen Link zur Creative Commons Lizenz beifügen und angeben, ob Änderungen vorgenommen wurden.

Die in diesem Kapitel enthaltenen Bilder und sonstiges Drittmaterial unterliegen ebenfalls der genannten Creative Commons Lizenz, sofern sich aus der Abbildungslegende nichts anderes ergibt. Sofern das betreffende Material nicht unter der genannten Creative Commons Lizenz steht und die betreffende Handlung nicht nach gesetzlichen Vorschriften erlaubt ist, ist für die oben aufgeführten Weiterverwendungen des Materials die Einwilligung des jeweiligen Rechteinhabers einzuholen.

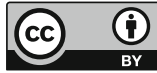

